\title{
Uma Análise Comparativa do Desempenho de Métodos Quase-Newton na Resolução de Problemas em Sísmica
}

L.F. DE MENDONÇA ${ }^{1}$, V.L.R. LOPES ${ }^{2}$, Departamento de Matemática Aplicada, IMECC, UNICAMP, 13083-970 Campinas, SP, Brasil.

\begin{abstract}
Resumo. Este trabalho descreve o problema de traçamento de raios, que é freqüente em Geofísica e introduz sua solução utilizando métodos quase-Newton. Na literatura em geral, problemas desse tipo eram sempre resolvidos utilizando o método de Newton. Aqui foram utilizados, além do método de Newton, os métodos quaseNewton: Broyden, Inverse Column Updating Method e Inverse $q$-Columns Updating Method. Uma análise comparativa do desempenho desses métodos é feita, traçando seus performance profiles usando como medidas o número de iterações e o tempo de execução.
\end{abstract}

\section{Introdução}

A resolução de um sistema de equações não lineares é uma tarefa necessária durante a resolução de problemas das mais diversas áreas (Física, Engenharia, Economia e outras Ciências) [12]. Para tanto, são utilizados métodos iterativos.

O método iterativo mais conhecido é o método de Newton, o qual possui convergência local quadrática. Entretanto, embora possuam convergência local superlinear (sob certas condições), os métodos quase-Newton constituem uma boa opção para a resolução de tais sistemas, pois (em geral) conseguem obter a solução em um tempo de execução menor [2], [3] e com menos esforço computacional.

Tal fato motiva este trabalho, o que descreve um problema freqüente em Geofísica (Sísmica) e o resolve utilizando o método de Newton e os seguintes métodos quase-Newton [10]: Broyden, Inverse Column Updating Method (ICUM) [9] e Inverse $q$-Columns Updating Methods [11], com $q=2$ (ICUM2) e $q=3$ (ICUM3). Por fim, é realizada uma análise dos resultados obtidos, seguindo a metodologia do traçamento do perfil de desempenho de cada método, introduzida por Dolan e Moré em 2002 (ver [5]). Mostramos graficamente parte das soluções. Os resultados obtidos nos levam à conclusão de que os métodos quase-Newton, em particular ICUM2, introduzido por Ferreira-Mendonça, Pérez e Lopes em [11], têm desempenho superior (e são mais baratos computacionalmente).

\footnotetext{
${ }^{1}$ E-mail: luziane@ime.unicamp.br; financiamento FAPESP (processo no. 2000-00375-4).

2E-mail: vlopes@ime.unicamp.br; financiamento FAPESP (processos no. 2001-04597-4 e no. 2001-07987-8).
} 


\section{Métodos Iterativos}

Nesta seção descrevemos os métodos iterativos que serão utilizados na resolução de nossas simulações. Tais métodos numéricos se aplicam quando estamos interessados em resolver um sistema de equações não lineares, representado por:

$$
F(x)=\left(f_{1}(x), f_{2}(x), \ldots, f_{n}(x)\right)^{T}=0, \quad F: \mathbb{R}^{n} \rightarrow \mathbb{R}^{n}, \quad F \in C^{1}\left(\mathbb{R}^{n}\right) .
$$

Denotamos por $J(x)$ a matriz Jacobiana da função $F$ aplicada no ponto $x$.

O método mais conhecido é o método de Newton, no qual a solução $x^{*}$ é aproximada por uma sequência de pontos $\left\{x^{k}\right\}$ gerada por:

$$
J\left(x^{k}\right) s^{k}=-F\left(x^{k}\right), \quad x^{k+1}=x^{k}+s^{k} .
$$

Os métodos quase-Newton são outra opção para resolver $F(x)=0$ e têm como proposta inicial evitar o cálculo de $J\left(x_{k}\right)$ em cada iteração (aproximando-a por uma matriz $B_{k}$ ), reduzindo assim o número de operações realizadas por iteração, e tentando ser "tão bons" quanto o método de Newton (no sentido de manter ao máximo as propriedades de convergência deste) [4].

Nesses métodos, a seqüência $\left\{x_{k}\right\}$ é gerada através da fórmula:

$$
B_{k} s^{k}=-F\left(x^{k}\right), \quad x^{k+1}=x^{k}+s^{k} .
$$

Uma classe de métodos quase-Newton bem sucedida é a dos métodos secantes. Neste caso, as matrizes de aproximação para a matriz Jacobiana são escolhidas de modo a satisfazer a "equação secante" $B_{k+1} s^{k}=y^{k}$, onde $y^{k}=F\left(x^{k+1}\right)-F\left(x^{k}\right)$.

O método de Broyden é um método quase-Newton secante, cuja fórmula para $B_{k+1}$ consiste numa correção de posto um sobre a matriz $B_{k}$; em particular, $B_{k+1}$ é a projeção ortogonal de $B_{k}$ no conjunto das matrizes que satisfazem a equação secante (considerando a norma de Frobenius). Pode-se também calcular $x^{k+1}=$ $x^{k}-B_{k}^{-1}\left(F\left(x^{k}\right)\right)$ diretamente, onde $B_{k+1}^{-1}$ é obtida a partir de $B_{k}^{-1}$ por meio de uma correção de posto um, fazendo uso da fórmula de Sherman-Morrison [6].

No método Inverse Column Updating Method (ICUM), apenas uma coluna da matriz de aproximação da inversa da matriz Jacobiana é atualizada a cada iteração, de modo que a equação secante sempre seja satisfeita [8], [9]. Em nossas implementações, o índice $j_{k}$ da coluna de $H_{k}=B_{k}^{-1}$ que deverá ser modificada, foi escolhido como $\left|y_{j_{k}}^{k}\right|=\left\|y^{k}\right\|_{\infty}$.

A nova matriz $H_{k+1}$ pode ser obtida através da equação

$$
H_{k+1}=H_{k}+\frac{\left(s^{k}-H_{k} y^{k}\right) e_{j_{k}}^{T}}{e_{j_{k}}^{T} y^{k}} .
$$

No método Inverse $q$-Columns Updating Method ([10], [11]), utilizamos $q=2$ (ICUM2) e $q=3$ (ICUM3) para determinar as trajetórias dos raios. Tal método atualiza $q$ colunas da matriz Jacobiana inversa, ou seja, $H_{k}$ é atualizada de tal forma 
que $H_{k+1}$ difira da matriz anterior em $q$ colunas, e satisfaça as $q$ últimas equações secantes.

Para o caso $q=2$, a fórmula de atualização da matriz $H_{k}$ é dada por:

$$
\begin{aligned}
H_{k+1}=H_{k}+\left(\frac{\left(e_{i_{2}}^{T} y^{k-1}\right) v_{1}^{k}-\left(e_{i_{2}}^{T} y^{k}\right) v_{2}^{k}}{\sigma^{k}}\right) e_{i_{1}}^{T} \\
+\left(\frac{\left(e_{i_{1}}^{T} y^{k}\right) v_{2}^{k}-\left(e_{i_{1}}^{T} y^{k-1}\right) v_{1}^{k}}{\sigma^{k}}\right) e_{i_{2}}^{T},
\end{aligned}
$$

onde temos $\sigma^{k}=\left(e_{i_{1}}^{T} y^{k}\right)\left(e_{i_{2}}^{T} y^{k-1}\right)-\left(e_{i_{2}}^{T} y^{k}\right)\left(e_{i_{1}}^{T} y^{k-1}\right), \quad v_{1}^{k}=s^{k}-H_{k} y^{k} \quad \mathrm{e}$ $v_{2}^{k}=s^{k-1}-H_{k} y^{k-1}$.

A escolha dos índices $i_{1}$ e $i_{2}$ das colunas a serem modificadas está sujeita à hipótese $\sigma^{k} \neq 0$. Adotamos inicialmente $\left|y_{i_{1}}^{k}\right|=\left\|y^{k}\right\|_{\infty} \mathrm{e}\left|y_{i_{2}}^{k-1}\right|=\left\|y^{k-1}\right\|_{\infty} ;$ quando $\left|\sigma_{k}\right|<10^{-6}$, alteramos o índice $i_{2}$, que passa a ser o índice que fornece o maior valor (em módulo) para $\sigma_{k}, \operatorname{com} i_{1}$ fixo, como anteriormente:

$$
\left|\left(\left(e_{i_{1}}^{T} y^{k}\right) y^{k-1}-\left(e_{i_{1}}^{T} y^{k-1}\right) y^{k}\right)_{i_{2}}\right|=\left\|\left(e_{i_{1}}^{T} y^{k}\right) y^{k-1}-\left(e_{i_{1}}^{T} y^{k-1}\right) y^{k}\right\|_{\infty}
$$

Tomando $q=3$, a fórmula de atualização da matriz $H_{k}$ será dada por uma correção de posto 3, sendo agora necessário escolher os três índices das colunas a serem modificadas $\left(i_{1}, i_{2}\right.$ e $\left.i_{3}\right)$, adotados previamente como $\left|y_{i_{1}}^{k}\right|=\left\|y^{k}\right\|_{\infty}$, $\left|y_{i_{2}}^{k-1}\right|=\left\|y^{k-1}\right\|_{\infty}$ e $\left|y_{i_{3}}^{k-2}\right|=\left\|y^{k-2}\right\|_{\infty}$. Tal escolha se altera quando o denominador da fórmula de atualização torna-se próximo de zero; neste caso, os índices $i_{2}$ e $i_{3}$, passam a ser os índices que fornecem o maior valor (em módulo) para o denominador, dado que $i_{1}$ está fixo ${ }^{3}$.

\section{O Problema}

Em pontos distintos da superfície da Terra (representada em duas dimensões), posicionam-se uma fonte de ondas sonoras $\mathbf{S}$ e um receptor (geofone) $\mathbf{G}$; a fonte emite uma onda sonora que atravessará um número determinado de regiões subterrâneas, e ao retornar à superfície, será captada pelo geofone. Tais regiões (perfeitamente elásticas, isotrópicas e homogêneas) compoem a estrutura da subsuperfície da Terra. O problema que estamos interessados em resolver consiste em determinar a trajetória de um raio $^{4}$ refletido/transmitido ao cruzar a subsuperfície [7].

As interfaces entre as regiões consecutivas são definidas em função da coordenada horizontal $x$; são contínuas e suaves (não será considerada intersecção entre as interfaces), e representadas por $z=f_{i}(x), i=1, \cdots, m$, onde $m$ corresponde ao número de interfaces e $z$ denota a coordenada vertical.

Denotaremos por $\left(a_{i}\right), \quad i=1, \cdots, m-1$, o número de vezes que o raio cruza (para baixo e para cima) a região situada entre as interfaces $f_{i}$ e $f_{i+1}$; na

\footnotetext{
${ }^{3}$ Para uma maior descrição desse método, veja [11].

${ }^{4} \mathrm{~A}$ frente de uma onda elementar pode ser descrita pela superfície $t=T(x, z)$, onde $T(x, z)$ é a função de tempo de trânsito. Os raios são trajetórias ortogonais ao movimento das frentes de onda e podem ser obtidos como pontos estacionários do funcional de Fermat, o qual representa o tempo de trânsito entre dois pontos dados [1].
} 
Figura 1 (a), $a_{1}=2$ e $a_{2}=1$, por exemplo. O vetor $a=\left(a_{1}, \cdots, a_{m-1}\right)$ é chamado de assinatura do raio. Como a assinatura não é suficiente para determinar de forma única a trajetória do raio, em nossos testes numéricos fixaremos que a trajetória do raio é tal que haja $2 a_{i}-1$ movimentos em cada região $i$ antes de passar para a região $i+1$; após essas reflexões, ele retorná diretamente ao geofone (Figura 1).

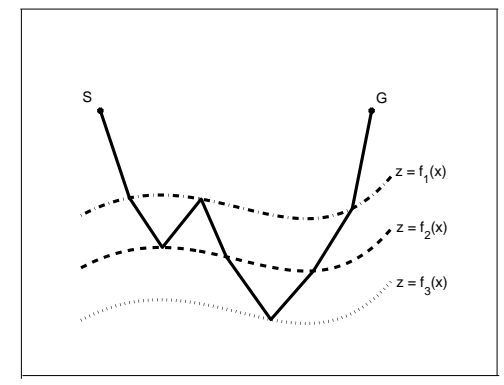

(a)

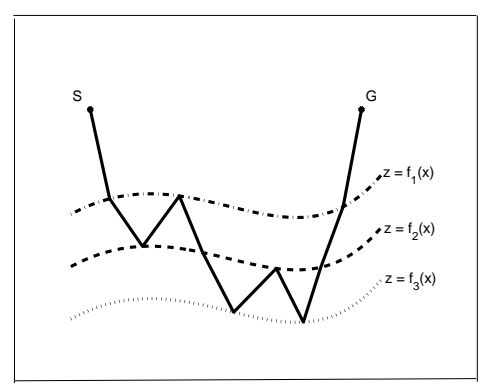

(b)

Figura 1: (a) - Raio com assinatura $(2,1)$; (b) - Raio com assinatura $(2,2)$.

Sob essas condições, os raios devem obedecer à lei de Snell (e como conseqüência, descrevem uma trajetória em linha reta em cada camada). Então, dados os pontos inicial $\mathbf{S}$ e final $\mathbf{G}$ na superfície da Terra, a velocidade da j-ésima região $\left(v_{j}\right)$, $j=1, \cdots, m$ e a assinatura $\left(a_{j}\right), j=1, \cdots, m-1$, o problema consiste em encontrar os pontos $\mathbf{X}_{\mathbf{k}}=\left(x_{k}, f_{i_{k}}\left(x_{k}\right)\right)$, para $k=1,2, \cdots, n$, que satisfazem a lei de Snell em cada refletor $i_{k}\left(\operatorname{adotaremos} \mathbf{X}_{\mathbf{0}}=\mathbf{S}\right.$ e $\left.\mathbf{X}_{\mathbf{n}+\mathbf{1}}=\mathbf{G}\right)$. A dimensão $n$ é obtida por $n=2 \sum_{j=1}^{m-1} a_{j}+1$.

Definindo $\tau_{k}=\left(1, f_{i_{k}}^{\prime}\left(x_{k}\right)\right)$ como o vetor tangente à k-ésima interface no ponto $\mathbf{X}_{\mathbf{k}}$, podemos escrever a lei de Snell como

$$
\frac{1}{v_{i_{k}}} \frac{\tau_{k}^{T}\left(\mathbf{X}_{\mathbf{k}}-\mathbf{X}_{\mathbf{k}-\mathbf{1}}\right)}{\left\|\tau_{k}\right\|\left\|\mathbf{X}_{\mathbf{k}}-\mathbf{X}_{\mathbf{k}-\mathbf{1}}\right\|}=\frac{1}{v_{i_{k+1}}} \frac{\tau_{k}^{T}\left(\mathbf{X}_{\mathbf{k}+\mathbf{1}}-\mathbf{X}_{\mathbf{k}}\right)}{\left\|\tau_{k}\right\|\left\|\mathbf{X}_{\mathbf{k}+\mathbf{1}}-\mathbf{X}_{\mathbf{k}}\right\|},
$$

onde $\|\cdot\|$ denota a norma euclidiana. Assim, utilizando a equação (3.1) para $k=1, \cdots, n$, obtemos um sistema não linear de $n$ equações e $n$ incógnitas, dado por

$$
\boldsymbol{\Phi}(\mathbf{x})=0
$$

$\operatorname{com} \boldsymbol{\Phi}: \mathbb{R}^{n} \rightarrow \mathbb{R}^{n}, \boldsymbol{\Phi}(\mathbf{x})=\left(\phi_{1}(\mathbf{x}), \phi_{2}(\mathbf{x}), \cdots, \phi_{n}(\mathbf{x})\right)^{T}$ e as funções $\phi_{k}: \mathbb{R}^{n} \rightarrow \mathbb{R}$ $(k=1, \cdots, n)$ definidas por

$$
\begin{aligned}
\phi_{k}(\mathbf{x})=v_{i_{k+1}} \frac{\left(x_{k}-x_{k-1}\right)+f_{i_{k}}^{\prime}\left(x_{k}\right)\left(f_{i_{k}}\left(x_{k}\right)-f_{i_{k-1}}\left(x_{k-1}\right)\right)}{\left[\left(x_{k}-x_{k-1}\right)^{2}+\left(f_{i_{k}}\left(x_{k}\right)-f_{i_{k-1}}\left(x_{k-1}\right)\right)^{2}\right]^{1 / 2}} \\
-v_{i_{k}} \frac{\left(x_{k+1}-x_{k}\right)+f_{i_{k}}^{\prime}\left(x_{k}\right)\left(f_{i_{k+1}}\left(x_{k+1}\right)-f_{i_{k}}\left(x_{k}\right)\right)}{\left[\left(x_{k+1}-x_{k}\right)^{2}+\left(f_{i_{k}}\left(x_{k+1}\right)-f_{i_{k}}\left(x_{k}\right)\right)^{2}\right]^{1 / 2}} .
\end{aligned}
$$




\section{Testes Numéricos}

A primeira estrutura utilizada é retratada na Figura 2 (a), com a fonte e o geofone posicionados na superfície de forma simétrica, a $1.5 \mathrm{~km}$ da origem. As interfaces são dadas por $z=f_{1}(x)=2-\exp \left(-10 x^{2}\right)$ e $z=f_{2}(x)=3.5-\exp \left(-10 x^{2}\right)$, onde $x$ representa a distância $(\mathrm{em} \mathrm{km})$; e as velocidades das regiões são, respectivamente $v_{1}=2 \mathrm{~km} / \mathrm{s}$ e $v_{2}=2.5 \mathrm{~km} / \mathrm{s}$.

Nessa estrutura, foram utilizadas as assinaturas $a=(3), a=(4)$ e $a=(5)$, as quais descrevem as trajetórias representadas na Figura 2 (b)-(d). Os pontos iniciais são, respectivamente

$$
\begin{aligned}
& \mathrm{x}^{\mathbf{0}}=(-1 ;-0.7 ;-0.4 ; 0 ; 0.4 ; 0.7 ; 1) \\
& \mathrm{x}^{\mathbf{0}}=(-1.35 ;-0.8 ;-0.4 ;-0.1 ; 0 ; 0.1 ; 0.4 ; 0.8 ; 1.35) \mathrm{e} \\
& \mathrm{x}^{\mathbf{0}}=(-1.3 ;-0.85 ;-0.5 ;-0.1 ;-0.05 ; 0 ; 0.05 ; 0.1 ; 0.5 ; 0.85 ; 1.3) .
\end{aligned}
$$

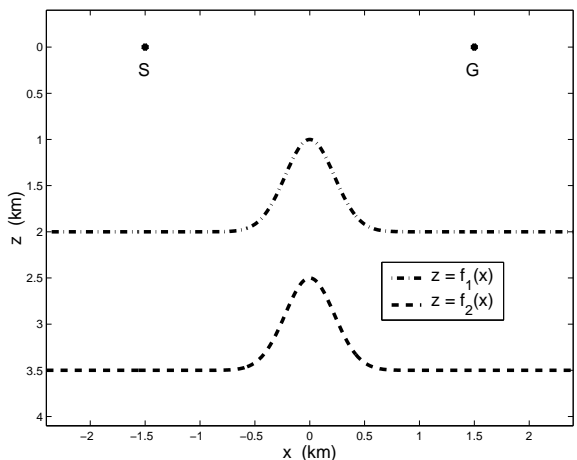

(a)

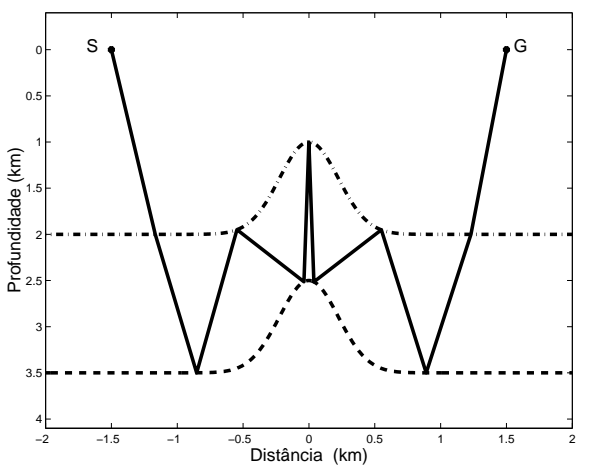

(c)

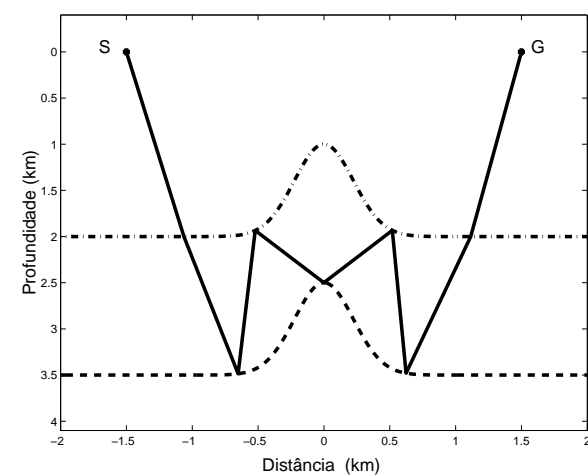

(b)

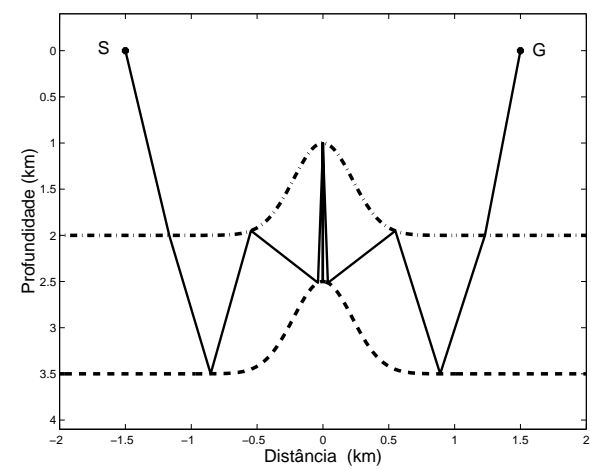

(d)

Figura 2: (a) Estrutura 1; (b) Estrutura 1 - $a=(3)$; (c) Estrutura 1 - $a=(4)$; (d) Estrutura $1-a=(5)$.

A segunda estrutura é dada na Figura 3 (a), com a fonte e o geofone dispostos 
da mesma forma que na estrutura anterior. As interfaces são

$$
f_{1}(x)=0.75+0.125 x, \quad f_{2}(x)=2-0.5 \exp \left(-2 x^{2}\right) \text { e } f_{3}(x)=3-x^{2} / 8,
$$

e as velocidades das regiões são $v_{1}=1.5 \mathrm{~km} / \mathrm{s}, v_{2}=2 \mathrm{~km} / \mathrm{s}$ e $v_{3}=2.5 \mathrm{~km} / \mathrm{s}$.

As assinaturas executadas pelo raio nesta estrutura foram $a=(1,1), a=$ $(2,1), a=(1,2)$ e $a=(2,2)$, cujos respectivos trajetos estão representados na Figura 3 (b)-(e). Os pontos iniciais são dados por

$$
\begin{aligned}
& \mathbf{x}^{\mathbf{0}}=(-1 ;-0.5 ; 0 ; 0.5 ; 1) \\
& \mathbf{x}^{\mathbf{0}}=(-1.3 ;-0.2 ; 0 ; 1.2 ; 2 ; 1.8 ; 1.8) \\
& \mathbf{x}^{\mathbf{0}}=(-1.125 ;-0.75 ;-0.375 ; 0 ; 0.375 ; 0.75 ; 1.125) \mathrm{e} \\
& \mathbf{x}^{\mathbf{0}}=(-1.3 ;-0.3 ; 0 ; 0 ;-0.3 ;-0.3 ; 0.8 ; 1.1 ; 1.8)
\end{aligned}
$$

respectivamente.

A última estrutura é dada na Figura 3 (f), com a fonte e o geofone dispostos da forma simétrica, a $80 \mathrm{~km}$ da origem. As interfaces são $z=f_{1}(x)=2.5$ e $z=f_{2}(x)=$ 4 , isto é, são linhas retas para facilitar a obtenção de um bom ponto inicial para a execução dos métodos numéricos. As velocidades nas regiões são $v_{1}=2.8 \mathrm{~km} / \mathrm{s}$ e $v_{2}=1.2 \mathrm{~km} / \mathrm{s}$.

Uma característica do problema de traçamento de raios em sísmica é ser muito sensível ao ponto inicial. É necessário que este ponto esteja suficientemente próximo da solução para que os métodos convirjam ${ }^{5}$. Devido à forma particular das interfaces utilizadas, tomaremos as componentes do ponto inicial como aquelas que dividem o intervalo $\left[x_{S}, x_{G}\right]$ em $n+1$ partes iguais, ou seja,

$$
\mathbf{x}_{\mathbf{k}}^{\mathbf{0}}=x_{S}+k\left(\frac{x_{G}-x_{S}}{n+1}\right), \quad k=1,2, \cdots, n .
$$

Testamos o traçamento de várias assinaturas nessa estrutura: $a=(70), a=$ (100), $a=$ (150), $a=$ (200), $a=$ (250), $a=$ (300), $a=$ (350), $a=$ (400), $a=$ (450) e $a=(500)$. Por gerarem sistemas de grande porte, além do números de iterações, também foi computado o tempo utilizado para a execução de cada método neste caso.

O método de Newton foi aquele que efetuou o menor número de iterações, como era de se esperar. Porém o tempo (medido em segundos) foi o maior de todos os métodos implementados. Os métodos quase-Newton (com exceção do método multi-coluna com $q=3$ ) conseguiram obter convergência de forma rápida (ver [12]); dentre eles, o ICUM2 foi o que apresentou o menor número de iterações e o ICUM o menor tempo. O método multi-coluna com $q=3$ demorou a convergir devido à dificuldade de escolha dos índices das colunas a serem alteradas.

O critério de convergência, para todos os métodos, é dado por $\left\|\Phi\left(x^{*}\right)\right\|_{\infty} \leq 10^{-5}$. A execução do algoritmo é interrompida quando o número de iterações excede 500 ou $\|\Phi(x)\|_{\infty} \geq 10^{20}$, quando dizemos que o método diverge.

\footnotetext{
${ }^{5} \mathrm{Em}[7]$, há a descrição de técnicas para encontrar um bom ponto inicial (é utilizado o método da continuação ou homotópico).
} 


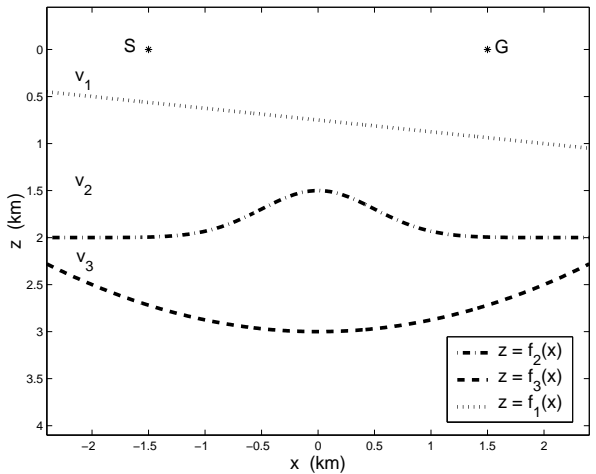

(a)

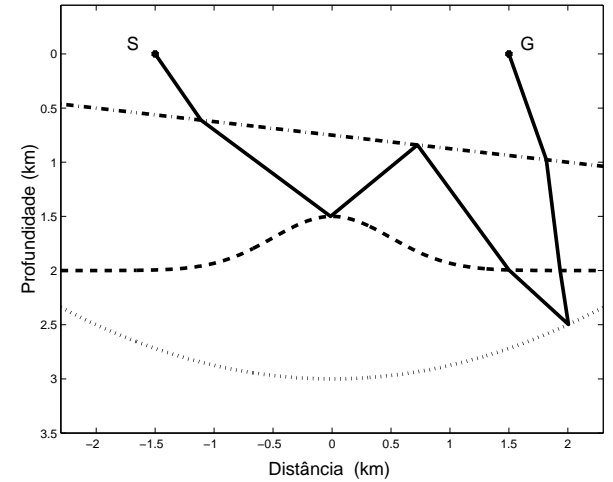

(c)

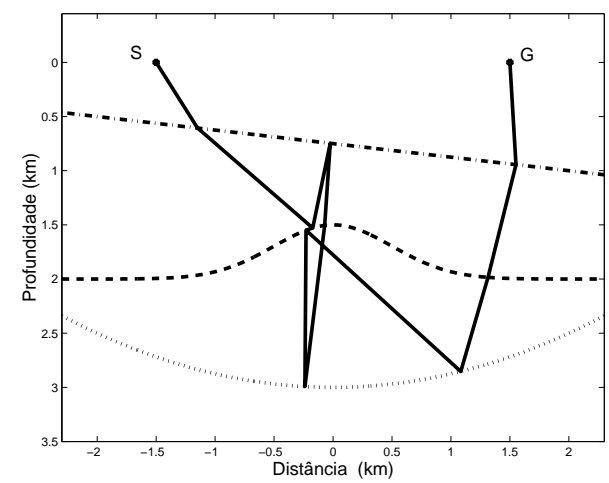

(e)

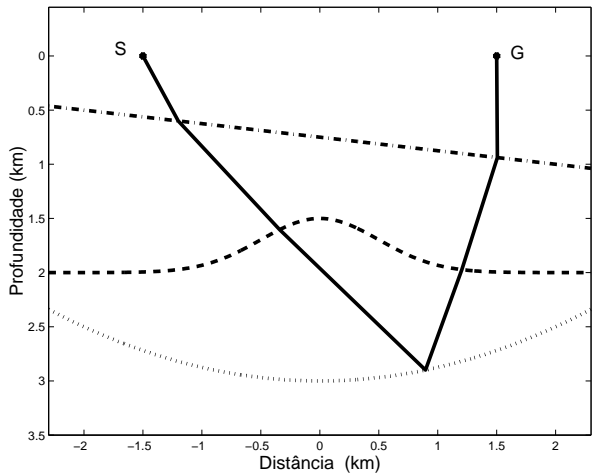

(b)

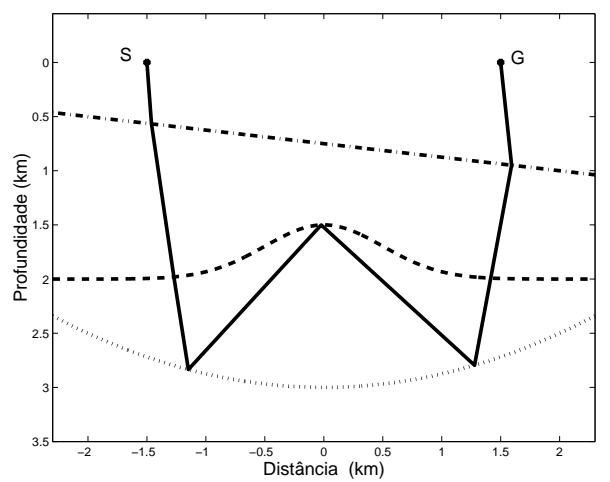

(d)

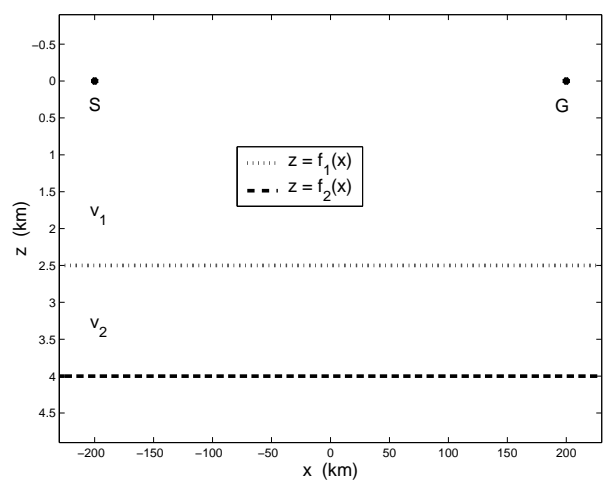

(f)

Figura 3: (a) Estrutura 2; (b) Estrutura $2-a=(1 ; 1)$; (c) Estrutura $2-a=(2 ; 1)$; (d) Estrutura $2-a=(1 ; 2)$; (e) Estrutura $2-a=(2 ; 2)$; (f) Estrutura 3. 
Com o intuito de visualizar e fazer uma análise comparativa entre o comportamento dos métodos, a Figura 4 representa o performance profile de tais métodos em relação ao número de iterações e ao tempo de execução, respectivamente (ver [5]).

O performance profile [5] compara o desempenho de $n_{s}$ métodos de um conjunto $S$ com relação à solução de $n_{p}$ problemas de um conjunto $P$, usando alguma medida de desempenho (número de iterações, tempo de CPU, etc.); $m_{s, p}$ denota o valor assumido pela medida para resolver o problema $p$ pelo método $s$. Para cada problema $p$ e método $s$, define-se a razão de performance $r_{s, p}$, que é dada por

$$
r_{s, p}=\frac{m_{s, p}}{\min \left\{m_{s, p}: s \in S\right\}}
$$

se o problema $p$ é resolvido pelo método $s\left(\rho_{s}(t) \geq 1\right)$; caso contrário, $r_{s, p}=r_{M}$, onde $r_{M}$ é um parâmetro fixo suficientemene grande.

A função de distribuição acumulativa $\rho_{s}: \mathbb{R} \rightarrow[0,1]$, para a razão de performance $r_{s, t}$ representa a performance total do método $s$ para resolver os problemas do conjunto $P$; ela é uma função não-decrescente e constante por partes. Tal função é definida como:

$$
\rho_{s}(t)=\frac{1}{n_{p}} \operatorname{size}\left\{p \in P \mid r_{s, p} \leq t\right\} .
$$

O valor de $\rho_{s}(1)$ indica a probabilidade do método $s$ ser o melhor método (dentre todos os pertencentes ao conjunto $S$ ), usando como medida de desempenho $m_{s, t}$. A eficiência do método $s$ em termos do número de problemas que podem ser resolvidos é observada por meio do valor mínimo de $\tau$, denotado por $\overline{\tau_{s}}$, tal que $\rho_{s}(\bar{\tau})=1$ para um valor $t<r_{M}$. Então, o método $\hat{s}$ será considerado o melhor em termos de eficiência quando $\overline{t_{\hat{s}}}=\min \left\{\bar{t}_{s}, \forall s \in S\right\}$.

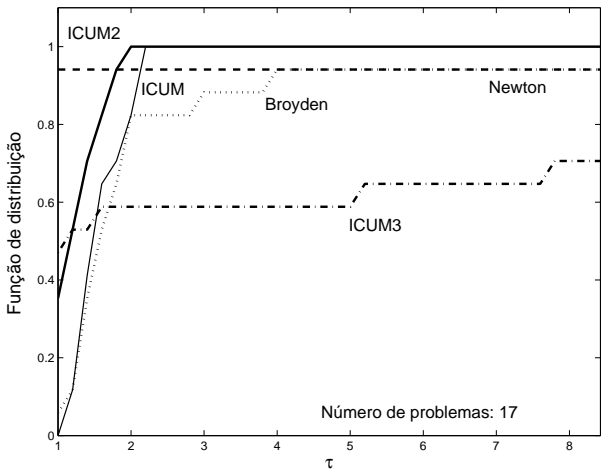

(a)

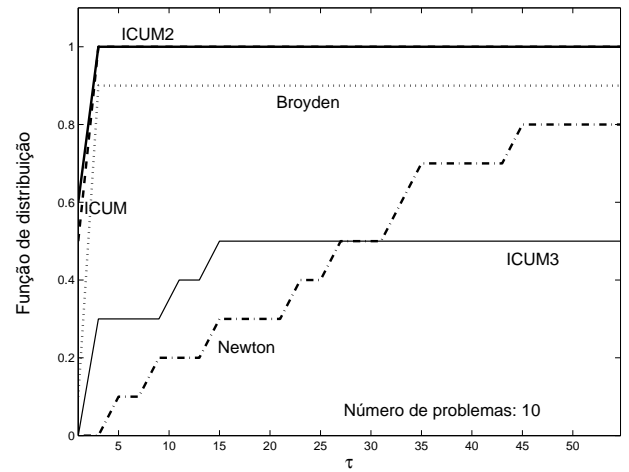

(b)

Figura 4: (a) Performance profile - número de iterações; (b) Performance profile - tempo de execução.

Os métodos de Newton e de Broyden não conseguiram resolver $6 \%$ do total de problemas propostos; nesse sentido, o ICUM3 foi aquele que apresentou pior desempenho, dado que conseguiu solucionar só $70 \%$ de todos os problemas propostos 
(Figura 4-(a)) e apenas 50\% dos problemas de grande porte (Figura 4-(b)). Observamos que os únicos métodos que obtiveram solução para todos os problemas foram ICUM e ICUM2.

Na Figura 4 (a), como já era esperado, vemos que o método de Newton realizou o menor número de iterações para a resolução de $94 \%$ dos problemas; entretanto, ele não conseguiu resolver os demais. O ICUM3 é o método quase-Newton que resolve o maior número de problemas $(50 \%)$ com o menor número de iterações; porém, ele obtém solução somente para $70 \%$ dos problemas propostos (o mesmo acontece com Broyden).

O ICUM resolve todos os problemas para $\tau \approx 2.2$, mas nenhum deles com o menor número de iterações. O ICUM2 pode ser considerado o método que obtém melhor desempenho (sua curva de desempenho mostra-se superior as demais, com exceção da Figura 4-(a), onde o método de Newton mostra-se superior no trecho inicial).

Na Figura 4, o pior desempenho dentre os métodos quase-Newton foi apresentado pelo ICUM3. Surpreendentemente, outro método que não obteve um bom desempenho foi o método de Newton pois, além de não resolver todos os problemas, ele necessita de uma grande quantidade tempo para convergir.

\title{
5. Conclusão
}

Conforme visto na seção anterior, uma boa forma de resolver o problema de traçamento de raios em Sísmica é utilizar métodos quase-Newton. De acordo com as Figura 4, o ICUM2 foi o método que apresentou melhor desempenho tanto em relação ao número de iterações quanto ao tempo necessário para convergência. Esse resultado vem reforçar a mesma conclusão obtida em [11], no qual Inverse $q-$ Column Updating Method foi apresentado e onde os mesmos métodos quase-Newton deste trabalho foram utilizados na resolução de outros problemas. Novamente, ICUM2 obteve o melhor desempenho.

\begin{abstract}
This work describes solutions to the Seismic Rays problem, which is frequently studied in Geophysics. In the literature, this type of problem is generally solved using the Newton method. In contrast, Quasi-Newton methods are used in this work. The performance of Newton's method is compared to that of the following quasi-Newton methods: Broyden, Inverse Column Updating and Inverse $q$-Columns Updating. This comparison is based on the performance profiles of the methods, where the number of iterations and CPU time were used as performance measures. As it will be shown, Quasi-Newton methods outperform Newton's method.
\end{abstract}

\section{Referências}

[1] N. Bleistein, "Mathematical methods for wave phenomena", Academic Press, 1984.

[2] C.G. Broyden, J.E. Dennis e J.J. Moré, On the local and superlinear convergence of quasi-Newton methods, J. Inst. Math. Appl., 12 (1973), 223-245. 
[3] J.E. Dennis e J.J. Moré, Quase-Newton methods, motivation and theory, SIAM Review, 19 (1997), 46-89.

[4] J.E. Dennis e R.B. Schnabel, "Numerical methods for unconstrained optimization and nonlinear equations", Prentice Hall, Englewood Cliffs, N.J, 1983.

[5] E.D. Dolan e J.J. Moré, Benchmarking optimization software with performance profiles, Math. Program. Ser., A91 (2002), 191-213.

[6] M.A. Gomes-Ruggiero, J.M. Martínez, e A.C. Moretti, Comparing algorithms for solving nonlinear systems of equations, SIAM Journal Scientific and Statistical Computing, 13, No. 2, 459-483.

[7] H.B. Keller e D.J. Perozzi, Fast seismic ray tracing, SIAM Journal of Applied Mathematics, 43, No. 4 (1983), 981-992.

[8] V.L.R. Lopes e J.M. Martínez, Convergence properties of the inverse columnupdating method, Optimization Methods and Software, 6 (1995), 127-144.

[9] J.M. Martínez e M.C. Zambaldi, An inverse column-updating method for solving large-scale nonlinear systems of equations, Optimization Methods and Software 1, 1 (1992), 129-140.

[10] L.F. de Mendonça, "Métodos de Atualização Multi-Coluna Inverso para Sistemas Não Lineares", Dissertação de Mestrado, Departamento de Matemática Aplicada, UNICAMP, Campinas, SP, 2002.

[11] L.F. de Mendonça, R. Pérez e V.L.R. Lopes, Inverse $q$-columns updating methods for solving nonlinear systems of equations, Journal of Computational and Applied Mathematics, 158 (2003), 317-337.

[12] R. Pérez e V.L.R. Lopes, Solving recent applications by quasi-Newton methods, Numerical Algorithms, 35 (2004), 261-285. 